\title{
Multi-trait linear reaction norm model to describe the pattern of phenotypic expression of some economic traits in beef cattle across a range of environments
}

\author{
Mário Luiz Santana Jr. • Joanir Pereira Eler • Annaiza Braga Bignardi • \\ Alberto Menéndez-Buxadera • Fernando Flores Cardoso • \\ José Bento Sterman Ferraz
}

Received: 17 April 2014 / Revised: 6 August 2014 / Accepted: 10 August 2014 /Published online: 21 September 2014

(C) Institute of Plant Genetics, Polish Academy of Sciences, Poznan 2014

\begin{abstract}
The multi-trait reaction norm (MTRN) model was extended to beef cattle reared under tropical conditions with the following objectives: to compare multi-trait (MT) and MTRN models regarding the genetic parameters obtained; and to characterize $\mathrm{G} \times \mathrm{E}$, the pattern of phenotypic expression, and the environmental sensitivity of animals for postweaning weight gain (PWG), scrotal circumference (SC), and annual average productivity of the cow (PRODAM). There was divergence in the estimates between the MT and MTRN models when the posterior probability intervals of additive genetic variances and heritability coefficients of PWG and PRODAM were analyzed. The MTRN model indicated an increase in heritability for PWG and PRODAM with improvement of the environmental conditions. For SC, heritability was practically the same, irrespective of the environmental conditions. The genetic correlations between the traits studied were low but varied over environments by the MTRN model.
\end{abstract}

M. L. Santana Jr. $(\bowtie) \cdot$ A. B. Bignardi

Grupo de Melhoramento Animal de Mato Grosso (GMAT), Instituto de Ciências Agrárias e Tecnológicas, Universidade Federal de Mato Grosso, Campus Universitário de Rondonópolis, MT-270, Km 06, 78735-901 Rondonópolis, MT, Brazil

e-mail: 10mario@gmail.com

J. P. Eler · J. B. S. Ferraz

Grupo de Melhoramento Animal e Biotecnologia (GMAB),

Departamento de Medicina Veterinária, Faculdade de Zootecnia e Engenharia de Alimentos, Universidade de São Paulo, C. Postal 23, 13635-970 Pirassununga, SP, Brazil

A. Menéndez-Buxadera

Departamento de Genética, Grupo Meragen, Rabanales,

Universidad de Córdoba, Cordoba 14071, Spain

F. F. Cardoso

Embrapa Pecuária Sul, BR-153, Km 603, C. Postal 242,

96401-970 Bagé, RS, Brazil
Considering genetic correlations obtained by the MTRN model for the same trait, lower estimates were obtained between extreme favorable and unfavorable environments. This finding suggest re-ranking of breeding values in different environments mainly for PWG and PRODAM. Thus, $\mathrm{G} \times \mathrm{E}$ is more important for PWG and PRODAM than for SC and should be included in the genetic evaluation of these traits. The traits PWG and PRODAM can be considered plastic traits, whereas $\mathrm{SC}$ is poorly plastic. The genetic trends in individual animal slopes indicate that the population is moving towards greater plasticity. This could be a matter of concern for breeders since greater plasticity seems to limit heritability and, consequently, the responses to selection.

Keywords Genetic parameter - Genotype by environment interaction · Nelore $\cdot$ Plasticity $\cdot$ Productive life $\cdot$ Tropical conditions

\section{Introduction}

The effect of genotype by environment interaction $(\mathrm{G} \times \mathrm{E})$ on animal performance has been commonly studied using multitrait (MT) models of the phenotypic expression of a trait in different environments (Falconer 1952) and, more recently, using linear reaction norm models, in which the phenotypic response of a genotype is described as a function of the environment (de Jong 1995). In general, when using the MT approach, $\mathrm{G} \times \mathrm{E}$ is of biological importance when the genetic correlation between the expression of the same trait in different environments differs from unity. According to the reaction norm approach, genetic variation in the slope of the reaction norm can be interpreted as the existence of $\mathrm{G} \times \mathrm{E}$ in the population (Pigliucci 2005). The reaction norm model permits to describe the evolution of a phenotype across a range of 
environments. Therefore, in contrast to the MT approach, which produces only point predictions, the reaction norm model permits the genetic prediction of an individual at any of the infinite points across a range of environments within the scale of values represented in the data set analyzed. In addition, reaction norm models can be useful to describe the environmental sensitivity of individuals, permitting the identification of those that are more sensitive or robust to environmental changes.

In view of the environmental heterogeneity found in tropical countries such as Brazil, there is strong evidence of the existence of $\mathrm{G} \times \mathrm{E}$ (Menéndez-Buxadera and Mandonnet 2006; Lopes et al. 2008; Cardoso and Tempelman 2012). Under these conditions, the response to selection may vary according to the environment where the animals are reared and selected. Logically, the use of genetic evaluation models that include $\mathrm{G} \times \mathrm{E}$ is justified in these cases. In this respect, a multi-trait reaction norm (MTRN) model might be useful to estimate genetic parameters according to environment, permitting simultaneous inferences on the responses to selection, pattern of phenotypic expression, and environmental sensitivity of animals for different traits.

The MTRN model has been little explored in animal breeding, particularly for beef cattle. Windig et al. $(2006,2011)$ applied MTRN models to production, health, and fertility traits of dairy cattle of European origin and demonstrated the usefulness of these models for the estimation of environmentdependent genetic correlations and the effects of selection on traits in different or same environments. It became clear that genetic correlations differ markedly depending on the environment. As a consequence, the response to selection in one environment is not the same in another for that population. In this respect, we extended MTRN models to beef cattle reared under tropical conditions with the following objectives: (1) to compare MT and MTRN models regarding the genetic parameters obtained, (2) to analyze genetic parameters according to environment, and (3) to characterize $\mathrm{G} \times \mathrm{E}$, the pattern of phenotypic expression, and the environmental sensitivity of animals for postweaning weight gain (PWG), scrotal circumference (SC), and annual average productivity of the cow (PRODAM).

\section{Materials and methods}

\section{Data}

The Nelore animals were born between 1980 and 2011 on 12 farms located in the Brazilian states of São Paulo, Mato Grosso do Sul, and Bahia (latitude -24.7 to -8.5 and longitude -57.9 to -37.5 ). The farms included in this study belong to the same company, which uses basic standard procedures of data collection and storage. The animals were kept on high-quality pasture (40\% Brachiaria brizantha, $50 \%$ Panicum maximum, and
$10 \%$ others) and received only salt and mineral supplementation. The breeding season, 90 days for heifers and 60 days for cows, ranged from October to January, depending on the beginning of the rainy season. Heifers were either inseminated $(\sim 40 \%)$ or multi-sire natural serviced ( $\sim 60 \%)$. The cow-to-bull ratio was about 35:1. Calves remained with their dams up to 7 months of age on high-quality pasture.

Traits

The PWG was defined as the weight gain from weaning (205 days) to 550 days of age, i.e., weight gain over a period of 345 days. The scrotal circumference was measured with an appropriate metric tape at yearling age (around 550 days of age). According to Santana et al. (2013a), PRODAM is defined as the weight (in $\mathrm{kg}$ ) of weaned calves produced annually by a cow during the time it stays in the herd. To obtain PRODAM, calf weaning weights were first adjusted using a model that included the fixed effect of the contemporary group (CG) (herd, year of birth, sex, and management group of the calf), age of calf at recording (linear effect) and age of dam at calving (linear and quadratic effects) as covariates, and the random effect of sire of the calf. For each cow, PRODAM was computed based on the adjusted weaning weight of the calf. Only cows that have been recorded since the beginning of their reproductive lives were included in this file. The following equation was used for the calculation of PRODAM:

$\operatorname{PRODAM}_{i}=\frac{\left(\sum_{j=1}^{n} W W_{i j}\right) * 365}{A C C_{n}-550}$,

where $P R O D A M_{i}$ is the average annual productivity, in $\mathrm{kg}$ of weaned calf per cow per year of productive life; $\sum_{i}^{n} W W_{i j}$ is the sum of the adjusted weaning weights of all calves of each cow; $W W_{i j}$ is the adjusted weaning weight of calf $j$ of cow $I$; and $A C C_{n}$ is the age of the cow (in days) at the last calving. The value 365 in the numerator corresponds to an annual production basis and the value 550 in the denominator refers to the first calving target at 30 months, with a minimum breeding age of 18 months. In recent years, heifers started to be exposed to bulls at 14 months of age (range: 1216 months). A second equation similar to Eq. 1 was used to calculate PRODAM for these heifers, with the value 365 in the denominator referring to the calving target at 24 months, with a minimum breeding age of 12 months.

For all traits, the records of animals in the CG with fewer than 20 animals, the $C G$ with all progeny of a single sire, sires with fewer than ten progeny records, records of animals with unknown sire or dam, and data exceeding 3.5 standard 
deviations above or below the mean of the respective $\mathrm{CG}$ were excluded (Table 1). All animals included in the present study had their own record of PWG.

\section{Environmental gradient}

The environmental gradients adopted were the CG solutions for PWG, which more comprehensively and reliably represented the conditions of management, climate, and location to which the animals of the present study were submitted. The CG solutions for PWG were obtained by applying an MT model to a data set that was similar to that used for genetic parameter estimation, but which included records of animals with unknown fathers.

Model and parameter estimation

The model for PWG and SC included the respective fixed effects of the contemporary group [farm, year of birth, sex (only PWG), postweaning management group] and the covariates age of animal at recording (linear effect) and age of dam at calving (linear and quadratic effects). For PWG, the model included the age of animal at weaning and age of animal at yearling as linear covariates. The statistical model for PRODAM included the fixed effect of the contemporary group (farm and year of birth). The MT model adopted can be described as:

$y_{i k}=\mu+a_{i}+$ fixed $_{k}+e_{i k}$,

where $y_{i k}$ is the record for the trait of animal $i$ of fixed effects $k ; \mu$ is the average performance over all animals; $a_{i}$ is the random additive genetic effect of animal $i$; ixed $_{k}$ is the set of fixed effects (CG, age of animal at recording, and age of dam at calving, as described above); and $e_{i k}$ is the random residual effect. The MTRN model can be described as follows:

$y_{i j k}=\mu+s_{F}+l_{i}+s_{i}, h_{j}+$ fixed $_{k}+e_{i j k}$,

where $y_{i j k}$ is the record for the trait of animal $i$ in environment $j$ of fixed effects $k ; \mu$ is the intercept for the fixed regression; $s_{F}$ is the fixed coefficient of a regression of $y$ on $h_{j}$ (environment $j$, defined to be solutions of the CG effect of PWG); $l_{i}$ is the random intercept (level) of the reaction norm of animal $i ; s_{i}, h_{j}$ is the random linear coefficient (slope) of a random regression of $y$ on $h_{j}$; fixed $_{k}$ is the set of fixed effects (as above); and $e_{i j k}$ is the random residual effect.

To obtain (co)variance components in the environmental gradient by MTRN, the genetic (co)variance matrix was computed as $\Phi K_{R N} \Phi^{\prime}$ :

$\Phi=\left[\begin{array}{cc}1 & s l, h 1 \\ 1 & s l, h 2 \\ \vdots & \vdots \\ 1 & s l, h j\end{array}\right]$

where $s l$ and $h_{j}$ are $j$ CG solutions that describe the environmental gradient (considered as a continuous variable expressed in a standardized form between -1 and +1 );

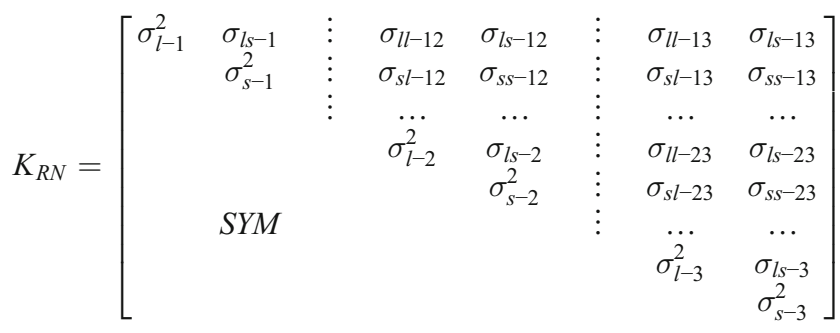

where $\sigma^{2}$ are the variances of level $(l)$ and slope $(s)$ and $\sigma$ are covariances between the level and slope of the traits studied $(1=$ PWG, $2=$ SC, $3=$ PRODAM $)$. Also,

$v\left[\begin{array}{l}G \\ R\end{array}\right]=\left[\begin{array}{cc}G=\phi\left[A \otimes K_{R N}\right] \phi^{\prime} & 0 \\ 0 & R=\left[\begin{array}{ccc}\sigma_{e 1}^{2} & \sigma_{e-12} & \sigma_{e-13} \\ \sigma_{e-12} & \sigma_{e 2}^{2} & 0 \\ \sigma_{e-13} & 0 & \sigma_{e 3}^{2}\end{array}\right] \otimes I\end{array}\right]$

where $G$ is the genetic (co)variance submatrix for level and slope; $A$ is the numerator of the relationship matrix among all
Table 1 Description of the data set for postweaning weight gain (PWG), scrotal circumference (SC), and average annual productivity of the cow (PRODAM) of Nelore cattle

\begin{tabular}{llll}
\hline & PWG $(\mathrm{kg})$ & SC $(\mathrm{cm})$ & PRODAM $(\mathrm{kg})$ \\
\hline Animals in the pedigree file & 133,892 & 83,364 & 47,386 \\
Animals with records & 78,709 & 35,939 & 21,674 \\
Number of sires with progeny record & 836 & 587 & 451 \\
Number of dams with progeny record & 44,139 & 24,174 & 16,240 \\
Number of sires with their own record & 366 & 280 & - \\
Number of dams with their own record & 12,931 & - & 4,587 \\
Number of contemporary groups (CG) & 1,612 & 749 & 88 \\
Mean number of records per CG & 48.827 & 47.982 & 246.295 \\
Mean of the trait & 117.210 & 27.895 & 129.190 \\
Standard deviation of the trait & 32.090 & 3.204 & 29.304 \\
\hline
\end{tabular}


animals; $R$ is the submatrix of the random residual effect with variance $\sigma_{e}^{2}$ and covariance $\sigma_{e}$ for the three traits studied; and $I$ is an identity matrix. The other terms are as described above. The (co)variance components were obtained by three-trait analysis under an animal model using Gibbs sampling. Analysis was performed with the GIBBS2F90 program (Misztal et al. 2002). The previous distributions for the (co)variance components were noninformative inverse Wishart distributions for all random effects. Analysis consisted of a single chain of 250,000 cycles, with a conservative burn-in period of 50,000 cycles and a thinning interval of 10 cycles. Thus, 20,000 samples were effectively used for final inferences. The $95 \%$ posterior probability interval (PPI95\%) was defined by the 2.5 th and 97.5 th percentiles of the posterior density.

Genetic merit

The estimated breeding value (EBV) of animal $i$ by the MTRN model was computed using the environmental gradient information as follows:

$E B V_{i}^{j}=\Phi_{(j)} \widehat{a}_{i}^{\prime}$,

where $\widehat{a}_{i}$ is the vector of estimated additive genetic values of the ordinary regression coefficients for animal $i$ (level and slope of the reaction norm) and $\Phi_{(j)}$ is a vector of ordinary coefficients evaluated in environment $j$ (CG solution for PWG).

Implications for selection

The top $5 \%$ of sires (with at least 25 progeny records) were selected for each trait using MT and MTRN models to determine the proportion of sires selected in common, i.e., the agreement of the sires selected between different points of the environmental gradient and the agreement of the sires selected by the MT and MTRN models. The EBVs obtained with the MTRN model were computed for favorable, intermediate, and unfavorable environments.

Measures to describe environmental sensitivity

Descriptive statistics of the individual slopes $(s)$ were computed for each trait. The difference between the EBV of a given animal in two environments for each trait was also calculated as a measure of plasticity: (EBV in a favorable environment - EBV in an unfavorable environment)/EBV in an unfavorable environment $\times 100$. The top 50 bulls (with at least 25 progeny records) for each trait by the MT model were sampled and then represented by their respective individual reaction norm obtained by the MTRN model . Genetic trends were estimated by regressing means (weighted by the number of animals) of $s$ of the animals by birth year (1996 to 2011).

\section{Results}

(Co)variance components and genetic parameters

In general, the CG solutions obtained in a previous analysis were positive for PWG (36.20 to $226.44 \mathrm{~kg}$ ), SC $(-0.98$ to $9.68 \mathrm{~cm})$, and PRODAM $(71.53$ to $157.05 \mathrm{~kg}$ ). The (co)variance components and genetic parameters obtained with the MT and MTRN models are shown in Tables 2 and 3. There was a greater divergence in the estimates between models when the PPI95\% of additive genetic variances and heritability coefficients of

Table 2 Components of (co)variance and genetic parameters for postweaning weight gain (PWG), scrotal circumference (SC), and average annual productivity of the cow (PRODAM) of Nelore cattle using a standard multi-trait model

\begin{tabular}{|c|c|c|c|}
\hline Item & Mean & $\mathrm{SD}$ & PPI95\% \\
\hline \multicolumn{4}{|c|}{ Additive genetic variance } \\
\hline PWG & 85.974 & 3.689 & $78.730 ; 93.230$ \\
\hline $\mathrm{SC}$ & 3.745 & 0.149 & $3.452 ; 4.034$ \\
\hline PRODAM & 112.187 & 13.272 & $87.855 ; 139.800$ \\
\hline \multicolumn{4}{|l|}{ Residual variance } \\
\hline PWG & 233.757 & 2.758 & $228.500 ; 239.100$ \\
\hline $\mathrm{SC}$ & 3.164 & 0.103 & $2.965 ; 3.369$ \\
\hline PRODAM & 664.575 & 11.839 & $641.200 ; 687.400$ \\
\hline \multicolumn{4}{|l|}{ Phenotypic variance } \\
\hline PWG & 319.731 & 1.998 & $315.770 ; 323.630$ \\
\hline $\mathrm{SC}$ & 6.909 & 0.069 & $6.773 ; 7.044$ \\
\hline PRODAM & 776.762 & 8.282 & $760.835 ; 793.200$ \\
\hline \multicolumn{4}{|l|}{ Heritability } \\
\hline PWG & 0.27 & 0.01 & $0.25 ; 0.29$ \\
\hline $\mathrm{SC}$ & 0.54 & 0.02 & $0.51 ; 0.57$ \\
\hline PRODAM & 0.14 & 0.02 & $0.11 ; 0.18$ \\
\hline \multicolumn{4}{|l|}{ Residual $^{2}$} \\
\hline PWG & 0.73 & 0.01 & $0.71 ; 0.75$ \\
\hline $\mathrm{SC}$ & 0.46 & 0.02 & $0.42 ; 0.49$ \\
\hline PRODAM & 0.86 & 0.02 & $0.82 ; 0.89$ \\
\hline \multicolumn{4}{|l|}{ Genetic correlation } \\
\hline $\mathrm{PWG}-\mathrm{SC}$ & 0.08 & 0.03 & $0.017 ; 0.13$ \\
\hline PWG - PRODAM & 0.20 & 0.05 & $0.10 ; 0.31$ \\
\hline SC - PRODAM & 0.11 & 0.05 & $0.01 ; 0.22$ \\
\hline \multicolumn{4}{|l|}{ Residual correlation } \\
\hline $\mathrm{PWG}-\mathrm{SC}$ & 0.35 & 0.01 & $0.33 ; 0.38$ \\
\hline PWG - PRODAM & 0.10 & 0.01 & $0.07 ; 0.12$ \\
\hline \multicolumn{4}{|l|}{ Phenotypic correlation } \\
\hline $\mathrm{PWG}-\mathrm{SC}$ & 0.23 & 0.07 & $0.22 ; 0.24$ \\
\hline PWG - PRODAM & 0.12 & 0.01 & $0.10 ; 0.13$ \\
\hline SC - PRODAM & 0.03 & 0.01 & $0.00 ; 0.06$ \\
\hline
\end{tabular}

$\mathrm{SD}=$ standard deviation; $\mathrm{PPI}=$ posterior probability interval Residual ${ }^{2}=$ residual variance as a proportion of the phenotypic variance 
Table 3 Components of (co)variance and genetic parameters for postweaning weight gain (PWG), scrotal circumference (SC), and average annual productivity of the cow (PRODAM) of Nelore cattle using multi-trait reaction norm model

\begin{tabular}{|c|c|c|c|c|c|c|c|}
\hline Item & Mean & $\mathrm{SD}$ & PPI95\% & Item & Mean & $\mathrm{SD}$ & PPI95\% \\
\hline \multicolumn{4}{|c|}{ Variance of level } & \multicolumn{4}{|l|}{ Heritability } \\
\hline PWG & 87.588 & 3.606 & $80.515 ; 94.900$ & PWG & 0.30 & 0.06 & $0.23 ; 0.43$ \\
\hline $\mathrm{SC}$ & 3.716 & 0.162 & $3.400 ; 4.032$ & $\mathrm{SC}$ & 0.56 & 0.02 & $0.51 ; 0.60$ \\
\hline PRODAM & 168.685 & 14.232 & $142.000 ; 198.400$ & PRODAM & 0.25 & 0.10 & $0.13 ; 0.46$ \\
\hline \multicolumn{4}{|c|}{ Variance of slope } & \multicolumn{4}{|l|}{ Slope $^{2}$} \\
\hline PWG & 33.278 & 4.314 & $25.125 ; 42.240$ & PWG & 0.10 & 0.02 & $0.07 ; 0.14$ \\
\hline $\mathrm{SC}$ & 0.460 & 0.090 & $0.309 ; 0.647$ & $\mathrm{SC}$ & 0.07 & 0.01 & $0.04 ; 0.09$ \\
\hline PRODAM & 153.036 & 24.078 & $112.100 ; 206.700$ & PRODAM & 0.18 & 0.04 & $0.12 ; 0.26$ \\
\hline \multicolumn{4}{|c|}{ Covariance between level and slope } & \multicolumn{4}{|l|}{ Residual $^{2}$} \\
\hline PWG & 23.330 & 1.735 & $20.050 ; 26.780$ & PWG & 0.70 & 0.06 & $0.57 ; 0.77$ \\
\hline $\mathrm{SC}$ & 0.001 & 0.064 & $-0.131 ; 0.127$ & $\mathrm{SC}$ & 0.44 & 0.02 & $0.40 ; 0.48$ \\
\hline PRODAM & 94.439 & 12.746 & $68.775 ; 118.300$ & PRODAM & 0.75 & 0.10 & $0.54 ; 0.87$ \\
\hline \multicolumn{4}{|c|}{ Additive genetic variance } & \multicolumn{4}{|l|}{ Genetic correlation } \\
\hline PWG & 99.790 & 30.616 & $66.330 ; 167.870$ & $\mathrm{PWG}-\mathrm{SC}$ & 0.11 & 0.04 & $0.03 ; 0.20$ \\
\hline $\mathrm{SC}$ & 3.884 & 0.236 & $3.469 ; 4.395$ & PWG - PRODAM & 0.17 & 0.12 & $-0.04 ; 0.39$ \\
\hline PRODAM & 224.798 & 127.328 & $96.852 ; 521.510$ & SC - PRODAM & 0.12 & 0.08 & $-0.07 ; 0.27$ \\
\hline \multicolumn{4}{|c|}{ Residual variance } & \multicolumn{4}{|l|}{ Residual correlation } \\
\hline PWG & 223.855 & 2.794 & $218.400 ; 229.300$ & $\mathrm{PWG}-\mathrm{SC}$ & 0.36 & 0.02 & $0.32 ; 0.39$ \\
\hline $\mathrm{SC}$ & 3.084 & 0.109 & $2.874 ; 3.300$ & PWG - PRODAM & 0.09 & 0.01 & $0.07 ; 0.12$ \\
\hline PRODAM & 623.333 & 11.199 & $600.700 ; 644.900$ & Phenotypic correlation & & & \\
\hline \multicolumn{4}{|c|}{ Phenotypic variance } & $\mathrm{PWG}-\mathrm{SC}$ & 0.24 & 0.01 & $0.22 ; 0.28$ \\
\hline PWG & 323.645 & 30.450 & $291.792 ; 391.160$ & PWG - PRODAM & 0.11 & 0.03 & $0.04 ; 0.15$ \\
\hline $\mathrm{SC}$ & 6.968 & 0.190 & $6.685 ; 7.405$ & SC - PRODAM & 0.05 & 0.04 & $-0.02 ; 0.12$ \\
\hline PRODAM & 848.131 & 126.731 & $723.048 ; 1143.040$ & & & & \\
\hline
\end{tabular}

$\mathrm{SD}=$ standard deviation; $\mathrm{PPI}=$ posterior probability interval; $\mathrm{Slope}^{2}=$ slope variance as a proportion of the phenotypic variance; Residual ${ }^{2}=$ residual variance as a proportion of the phenotypic variance

PWG and PRODAM were analyzed. The posterior means of heritability were low for PRODAM ( 0.14 to 0.25$)$, moderate for PWG (0.27 to 0.30), and high for SC (about 0.55 ). The MT model provided slightly lower posterior means for heritability estimates than the MTRN model. As can be seen in Fig. 1, the MTRN model indicated an increase in heritability for PWG and PRODAM with improvement of the environmental conditions. For SC, heritability was practically the same, irrespective of the environmental production conditions. Slightly lower heritability was observed in intermediate environments.

In general, the posterior means of genetic correlations between the traits studied were low (Tables 2 and 3). The posterior means of phenotypic and residual correlations were also low and did not exceed 0.36 . The correlations between level and slope for the same trait obtained with the MTRN model (Table 4) ranged from low to moderate magnitude (0 to 0.59 ). The correlations between the slopes of the different traits also ranged from low to moderate $(-0.06$ to 0.45$)$. However, the correlations between the levels of the different traits were even lower, ranging from 0.08 to 0.17 . The posterior means of correlations between level and slope of the different traits ranged from -0.22 to 0.20 .
Figure 2 shows the genetic correlations between traits and for the same trait obtained with the MTRN model across the

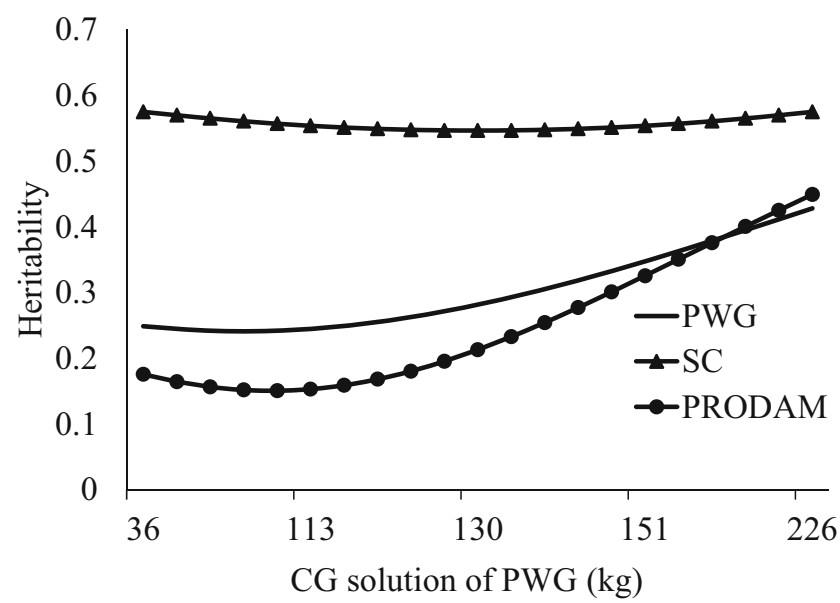

Fig. 1 Posterior means of heritability estimates according to the environmental gradient obtained by the multi-trait reaction norm (MTRN) model for postweaning weight gain (PWG), scrotal circumference (SC), and average annual productivity of the cow (PRODAM) of Nelore cattle 
Table 4 Posterior mean ( \pm standard deviation) of the correlation between level and slope of the reaction norm for postweaning weight gain (PWG), scrotal circumference (SC), and average annual productivity of the cow (PRODAM) of Nelore cattle using multi-trait reaction norm model

\begin{tabular}{|c|c|c|c|c|c|c|c|}
\hline \multirow[t]{2}{*}{ Trait } & \multirow[t]{2}{*}{ Parameter } & \multicolumn{2}{|l|}{ PWG } & \multicolumn{2}{|l|}{$\mathrm{SC}$} & \multicolumn{2}{|l|}{ PRODAM } \\
\hline & & Level & Slope & Level & Slope & Level & Slope \\
\hline \multirow[t]{2}{*}{ PWG } & Level & 1.000 & $0.434 \pm 0.037$ & $0.080 \pm 0.032$ & $0.084 \pm 0.094$ & $0.172 \pm 0.049$ & $0.053 \pm 0.072$ \\
\hline & Slope & & 1.000 & $-0.023 \pm 0.061$ & $0.451 \pm 0.102$ & $-0.226 \pm 0.094$ & $-0.067 \pm 0.151$ \\
\hline \multirow[t]{2}{*}{$\mathrm{SC}$} & Level & & & 1.000 & $0.000 \pm 0.049$ & $0.145 \pm 0.052$ & $0.205 \pm 0.093$ \\
\hline & Slope & & & & 1.000 & $-0.127 \pm 0.111$ & $0.049 \pm 0.150$ \\
\hline \multirow[t]{2}{*}{ PRODAM } & Level & & & & & 1.000 & $0.589 \pm 0.049$ \\
\hline & Slope & & & & & & 1.000 \\
\hline
\end{tabular}

environmental gradient. Considering genetic correlations for the same trait, lower estimates were obtained between extreme favorable and unfavorable environments ( 0.49 for PWG, 0.78 for SC, and 0.06 for PRODAM). Intermediate environments showed a higher correlation with favorable environments for PWG (0.91) and PRODAM (0.89), whereas for SC, the genetic correlation of intermediate environments with extreme favorable and unfavorable environments was essentially the same $(0.94)$. With respect to genetic correlations between different traits, the highest correlations were obtained between SC and PRODAM, reaching a value of 0.42 between favorable environments for SC and unfavorable environments for PRODAM. When intermediate or unfavorable environments for SC were analyzed, the genetic correlation between this trait and PRODAM was almost zero or even negative. The genetic correlation between PWG and PRODAM was about 0.3 when PRODAM in an unfavorable environment was considered. However, this correlation reached a value close to zero when PRODAM in an extreme favorable environment was considered. The genetic correlation between PWG and SC was higher in unfavorable environments (0.17). In contrast, the genetic correlation between these traits was -0.06 when opposite extreme environments were analyzed, for example, PWG in an unfavorable environment and $\mathrm{SC}$ in a favorable environment.

The eigendecomposition of the genetic correlation matrices of each trait showed that the first two eigenvalues explained 89.86 and $10.13 \%$ of the genetic variance in PWG, 95.80 and $4.19 \%$ of the variance in SC, and 80.52 and $19.45 \%$ of the variance in PRODAM, respectively. The corresponding first eigenfunction showed a relatively flat pattern for SC across the environmental gradient (Fig. 3). In contrast, for PWG and PRODAM, the corresponding first eigenfunction was slightly lower in unfavorable environments, maintaining a flat pattern
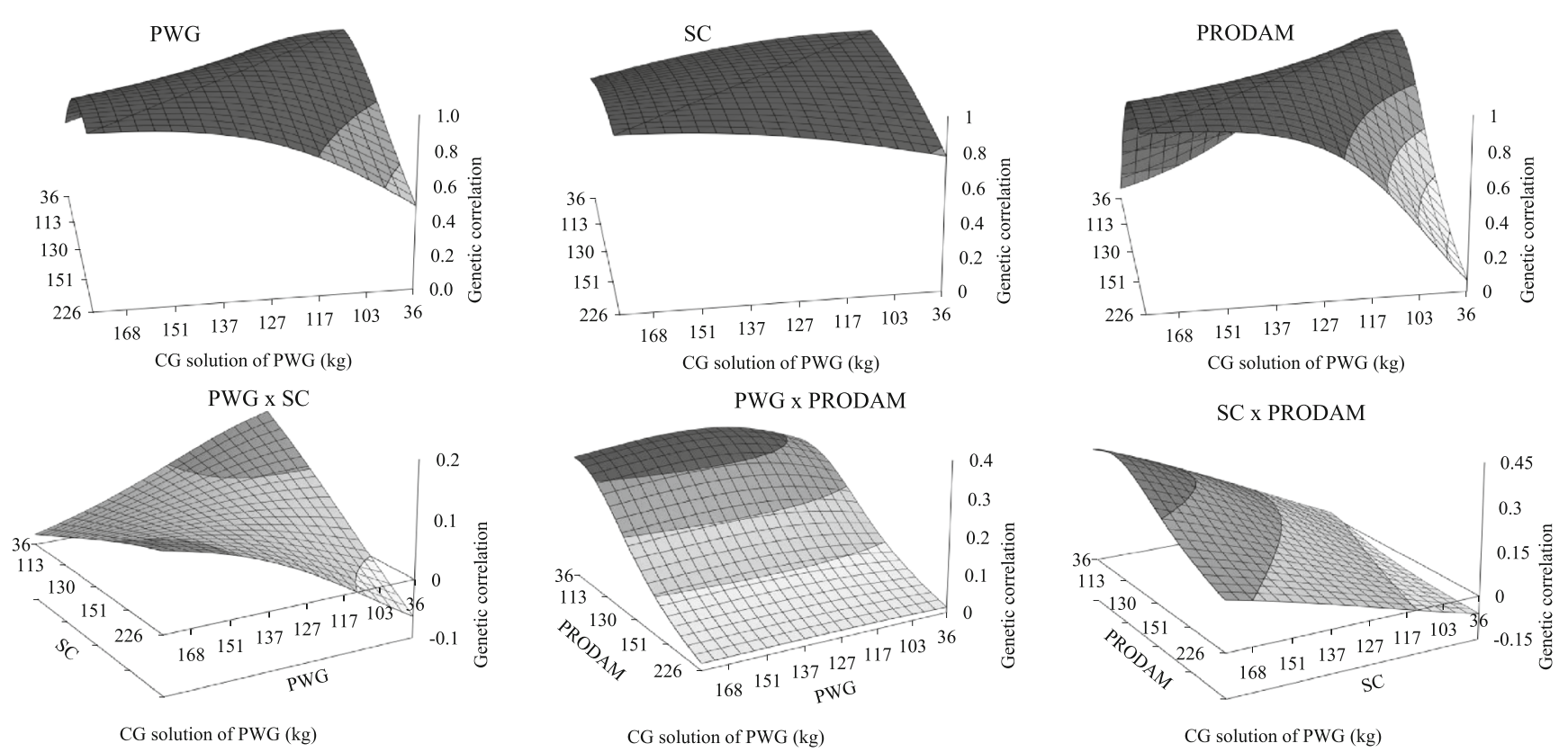

Fig. 2 Posterior means of genetic correlations according to the environmental gradient between traits and for the same trait of Nelore cattle by the multitrait reaction norm model. $\mathrm{PWG}=$ postweaning weight gain; $\mathrm{SC}=$ scrotal circumference; PRODAM=average annual productivity of the cow 

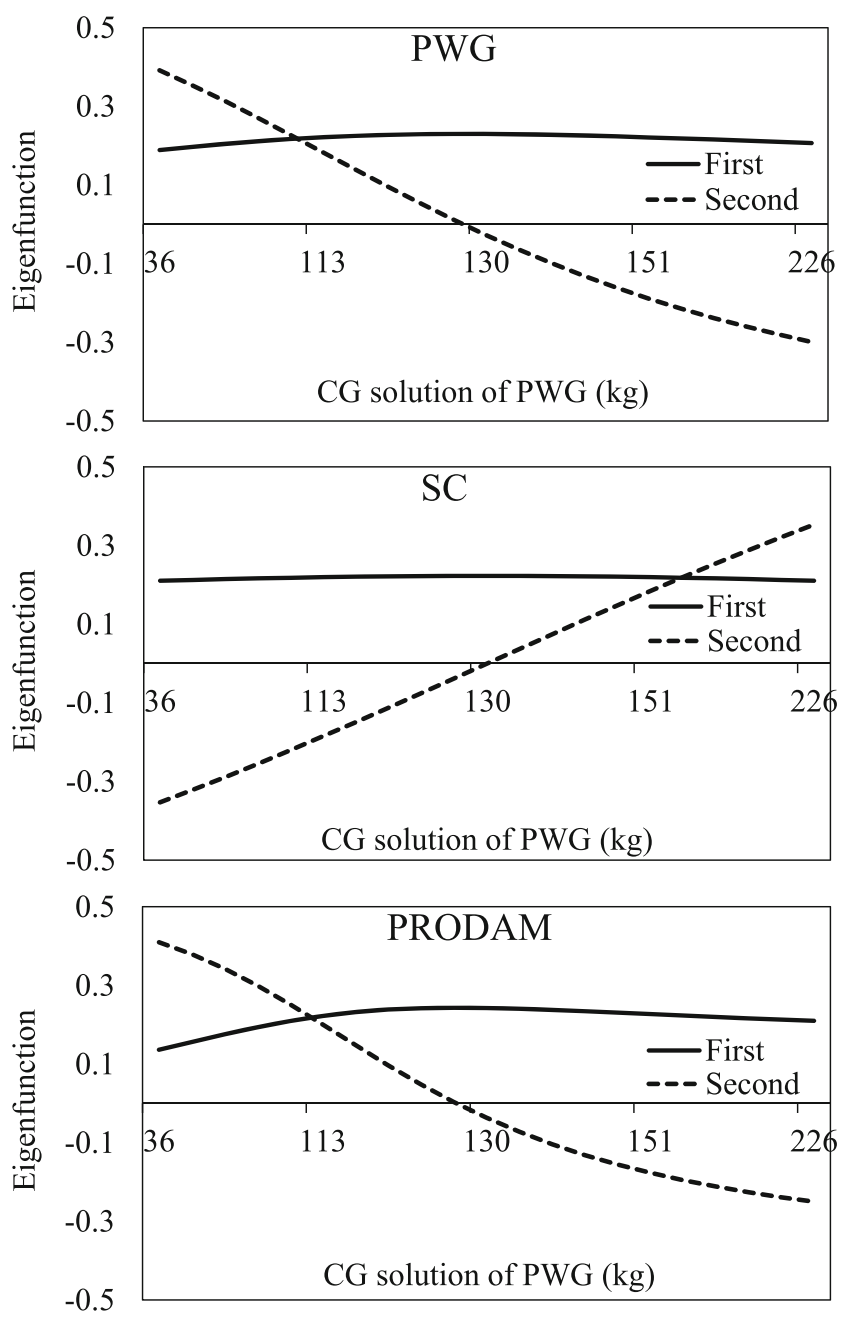

Fig. 3 Eigenfunctions associated to the genetic correlation matrix for postweaning weight gain (PWG), scrotal circumference (SC), and average annual productivity of the cow (PRODAM) of Nelore cattle according to the environmental gradient by the multi-trait reaction norm (MTRN) model

with improvement of environmental conditions. The corresponding second eigenfunction was not parallel to the horizontal axis of the environmental gradient for all traits studied.

\section{Environmental sensitivity}

The traits studied presented different plasticity patterns, as shown in Table 5. The individual slopes were more dispersed for PRODAM, as demonstrated by a higher coefficient of variation. Skewness was positive for the distribution of individual slopes for all traits (long tail on the right). Kurtosis was notably greater for PRODAM than for PWG or SC, demonstrating differences in the environmental sensitivity of each trait in the population studied. The genetic trend in individual slopes was negative for $\mathrm{SC}$, indicating an increase in the proportion of animals that do not respond favorably to improvements of environmental conditions for this trait (Table 5). On the basis of the positive genetic trend in the slope of PWG and PRODAM and the respective annual slope averages (data not shown), a significant increase was observed in the proportion of plastic animals that respond well to improvements of environmental conditions. According to the measure of plasticity adopted (mean percent alteration in the reaction norm), PRODAM was the most plastic trait and SC the least plastic. Therefore, much greater oscillations in breeding values of animals are expected for PRODAM than for SC across the environmental gradient. As illustrated in Fig. 4, the crossing of reaction norms of sires occurred, to a greater or lesser extent, for all traits studied; however, reaction norms were less dispersed for SC compared to PWG and PRODAM.

\section{Rank changes}

When the top $5 \%$ of sires were selected for PWG, SC, and PRODAM in each environment (unfavorable, intermediate, and favorable) using the MTRN model, the proportions of sires selected in common were different from unity for all traits, especially for PWG and PRODAM (values of up to 0.29) (Table 6). Comparison of the top $5 \%$ of sires selected by MT analysis and the top $5 \%$ sires of selected by the MTRN model in each environment showed important discordance in the selection for PWG and PRODAM in extreme environments.

\section{Discussion}

The CG solutions were positive for PWG. This finding is expected, since the animals of the present Nelore population belong to the same company, which generally adopts standardized management practices. In contrast, Corrêa et al. (2009) reported CG solutions for PWG ranging from -73.6 to $243.1 \mathrm{~kg}$ for Brazilian Devon cattle. Cardoso and Tempelman (2012) obtained CG solutions for PWG ranging from -92.6 to $265.5 \mathrm{~kg}$ for Brazilian Angus cattle.

It is reasonable to consider the heterogeneity of variances, particularly for PWG and PRODAM, since wider PPI95\% for additive genetic variance and heritability were obtained with the MTRN than with the MT model (Tables 2 and 3). The heterogeneity of additive genetic variance has also been reported by Pégolo et al. (2009), who studied weight at 450 days of age in Nelore cattle using a reaction norm model. Important slope variance was observed for all traits studied, especially for $\mathrm{PWG}$, demonstrating $\mathrm{G} \times \mathrm{E}$ across the environmental gradient. Based on the fact that slope variance is given as a proportion of phenotypic variance, a greater response to selection can be expected for the environmental sensitivity of PRODAM.

High heritability estimates were obtained for SC, which has commonly been reported as a highly heritable trait in Nelore cattle (Eler et al. 2004). The heritabilities for PWG and 
Table 5 Summary statistics of the individual slopes $(s)$ for postweaning weight gain (PWG), scrotal circumference (SC), and average annual productivity of the cow (PRODAM) of Nelore cattle using multi-trait reaction norm model

AGC $=$ annual genetic changes from 1996 to 2011; $\mathrm{SE}=$ standard error; $* * *$ Trend significantly different from $0(P<0.0001)$

\begin{tabular}{llll}
\hline Item & \multicolumn{2}{l}{ Traits } & \\
\cline { 2 - 4 } & PWG (kg) & SC (cm) & PRODAM (kg) \\
\hline Mean & 2.013 & 0.202 & 2.903 \\
Standard deviation & 2.240 & 0.200 & 3.666 \\
Coefficient of variation (\%) & 111.288 & 99.152 & 126.264 \\
Percentile 2.5 \% & -2.153 & -0.156 & -3.951 \\
Percentile 97.5 \% & 6.502 & 0.621 & 10.825 \\
Skewness & 0.132 & 0.232 & 0.284 \\
Kurtosis & 0.176 & 0.252 & 0.990 \\
AGC \pm SE & $0.091 \pm 0.014 * * *$ & $-0.005 \pm 0.001 * * *$ & $0.220 \pm 0.021 * * *$ \\
Average plasticity (high $\times$ low) $(\%)$ & 832.702 & 588.498 & $1,702.212$
\end{tabular}

PRODAM estimated with either the MT or MTRN models were similar to those reported in the literature $(0.15$ to 0.23$)$ (Eler et al. 2008; Santana et al. 2013a). For PWG and PRODAM analyzed with the MTRN model, heritability increased considerably with the improvement of environmental conditions (Fig. 1). Therefore, greater responses to selection of these traits can be expected in intermediate and favorable environments. Mattar et al. (2011), studying weight at 420 days of age in Canchim cattle (5/8 Charolais:3/8 Zebu), and Cardoso and Tempelman (2012), analyzing PWG in Brazilian Angus cattle, observed a similar trend in heritability using reaction norm models. Studies applying reaction norm models to estimate the genetic parameters of traits similar to PRODAM are sparse in the literature. Morales et al. (2013) applied a random regression model to weights at 120 and 180 days of age as a function of the number of calvings of a cow in Retinta cattle. The authors obtained markedly higher heritability estimates than those observed in the present study (0.30 to 0.60$)$. For $\mathrm{SC}$, the response to selection should be practically the same in any environment, since heritability remained basically unchanged across the environmental gradient. The heritabilities for SC were slightly lower in intermediate environments. A similar trend has been reported by Santana et al. (2013b), who used a reaction norm model to analyze SC in composite beef cattle in Brazil. This fact seems to be related to the greater crossing of individual reaction norms in the mid-region of the environmental gradient.

The posterior means of genetic correlations between the traits studied were low, especially between PWG and SC and between SC and PRODAM (Tables 2 and 3). Santana et al. (2013a) estimated posterior means of genetic correlations between PWG and SC that were higher than those obtained in the present study (0.23), and between SC and PRODAM that were similar to those observed here $(0.08)$ for the same Nelore population using a five-trait model. In this respect, selection for any of these traits should result in a small correlated response in the other traits. When the genetic correlations between traits across the environmental gradient are examined
(Fig. 2), it is clear that the responses to selection can vary considerably depending on the environment analyzed. Some genetic correlations of low magnitude became moderate, e.g., the correlation between $\mathrm{SC}$ in an unfavorable environment and PRODAM in a favorable environment $(-0.06)$ versus SC in a favorable environment and PRODAM in an unfavorable environment (0.42). The genetic correlations of the same trait in different environments were different from unity in favorable versus unfavorable environments (Fig. 2), implying that many of the same genes that influence PWG or PRODAM in favorable environments do not influence these traits in unfavorable environments. The same does not apply to SC. Lopes et al. (2008), studying Nelore cattle reared in southern Brazil, also reported genetic correlations below unity for the same trait in different environments and even negative correlations for weight at 205 days of age. Nephawe et al. (1999) obtained genetic correlations ranging from 0.50 to 0.97 for birth weight and from 0.32 to 0.97 for weaning weight of Bonsmara cattle reared in four ecological regions of South Africa.

The correlations between level and slope obtained with the MTRN model (Table 4) for the same trait were of low to moderate magnitude, indicating predominantly re-ranking of breeding values in different environments. Thus, the best animals in one environment are not necessarily the same in the other. The same observation was made by Santana et al. (2013b) based on a correlation between level and slope of 0.40 for weaning weight and of 0.14 for SC in composite beef cattle in Brazil. The posterior mean of the correlation between level and slope for SC was zero, i.e., selection for a higher level will basically lead to no response in the environmental sensitivity of the animals. The low and positive genetic correlations between trait levels indicate that selection for the level of one trait will not be effective in modifying the level of the other. In general, selection for the level of one trait should result in a small or no correlated response in the environmental sensitivity of other traits, since the estimates of correlation between level and slope of the reaction norm were close to zero. On the basis of the correlation between the slopes of different traits, selection 

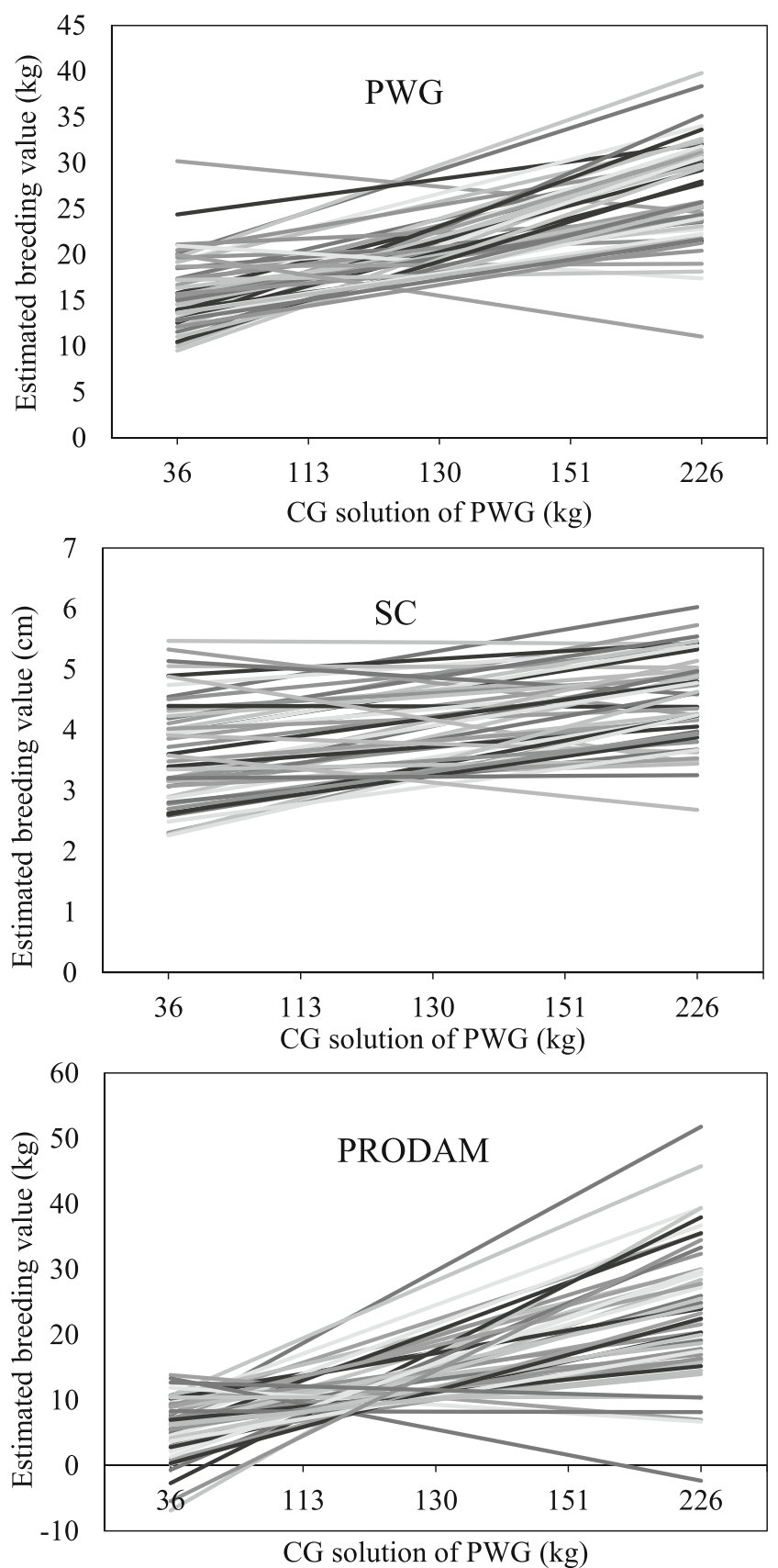

Fig. 4 Individual reaction norms according to the environmental gradient obtained by the multi-trait reaction norm model for 50 Nelore sires [top 50 by the standard multi-trait model] for postweaning weight gain (PWG), scrotal circumference (SC), and average annual productivity of the cow (PRODAM)

for the slope of one trait should result in a correlated response in the slope of the other (e.g., correlation of 0.45 between PWG and SC). Therefore, breeders should pay attention to these relationships which may have long-term implications, since Nelore breeding programs primarily favor animals that are less sensitive to environmental changes and which present a higher reaction norm level for the traits studied.

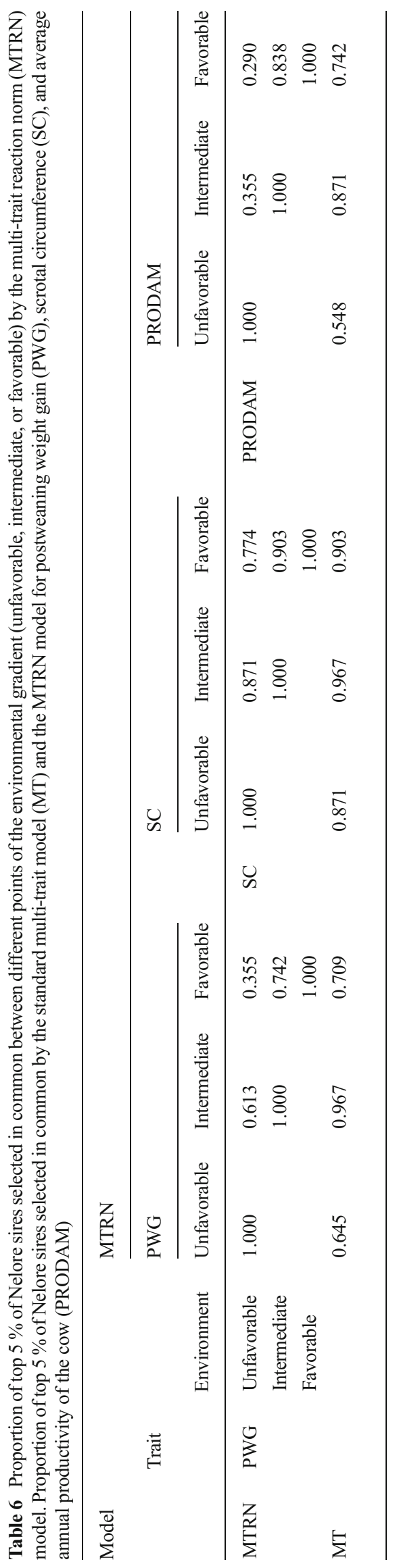


Most of the genetic variability observed in the performance of PWG, SC, and PRODAM was explained by the first eigenvalue associated with the genetic correlation matrix of each trait. The first eigenfunction corresponding to $\mathrm{SC}$ was flat across the environmental gradient, demonstrating that selection for this eigenfunction will result in the same response in all environments. For PWG and especially for PRODAM, selection for the first eigenfunction may not favor these traits in unfavorable or favorable environments. Alternatively, selection that favors the second eigenfunction could improve the performance of PWG and PRODAM in those environments. However, selection for the second eigenfunction would be less efficient, since only a small percentage of variability was explained by the second eigenvalue.

An important difference was observed in the distributions of individual animal slopes, as shown in Table 5. The distributions of individual slopes of all traits were leptokurtic (heavier tails and a higher peak than a normal distribution), particularly for PRODAM. For SC, the coefficient of variation and measure of plasticity adopted indicate less variation in the individual reaction norm slopes across the environmental gradient. As shown in Fig. 4, the reaction norms for SC are generally more parallel than those obtained for PWG or PRODAM. On the other hand, greater plasticity was observed for PWG and PRODAM. As a consequence, greater oscillations of breeding values across the environmental gradient and, logically, more intense re-ranking are expected for PWG and PRODAM. Tonsor et al. (2013), evaluating 13 traits of plants (Arabidopsis thaliana), found that plasticity was negatively associated with heritability. In this respect, more plastic traits exhibited, on average, $57 \%$ lower heritability than non-plastic traits. In the present study, the mean heritabilities for PWG and PRODAM were 50 and $73 \%$ (MT) and 46 and $55 \%$ (MTRN) lower than the heritability for SC, respectively. Taken together, these findings suggest that $\mathrm{SC}$ is poorly plastic, whereas PWG and PRODAM are plastic traits. The genetic trends in the slopes should serve as an alert to breeders since PWG and PRODAM are moving towards greater plasticity in the present population (Table 5). According to Tonsor et al. (2013), the cost of plasticity is a reduction in heritability and the response to selection of some of the traits studied here may, therefore, be compromised in the future. Kolmodin and Bijma (2004) suggested including the restriction of genetic changes in plasticity in the selection objectives in order to prevent animals from becoming excessively plastic. The plasticity of traits in the present population should, therefore, be taken into consideration during the selection process.

There was an important divergence in selection between the MT and MTRN models, especially for PWG and PRODAM in extreme environments (Table 6). This finding indicates the need for specific genetic evaluation in extreme environments for these traits. In comparison to the official procedure of genetic evaluation for the population studied
(MT), the MTRN model permits to obtain specific breeding values for each animal in each environment. In this case, each farm could use sires in a more rational manner. As can be seen in Fig. 4, a large part of the 50 best sires according to MT (official model) produces good responses in one environment and poor responses in others; however, when used, these animals transmit a sample of their entire genotype, a fact that may limit the genetic progress of the population. The MTRN model may, therefore, be more coherent from a technical and biological point of view for the genetic evaluation of the present Nelore population and could be recommended to meet the interests of both producers and technicians.

\section{Conclusion}

Differences exist between the multi-trait (MT) and multi-trait reaction norm (MTRN) models in terms of selection in extreme environments, particularly for postweaning weight gain (PWG) and annual average productivity of the cow (PRODAM). The advantage of the MTRN model is that it permits to obtain specific breeding values for each environment, exactly by considering that genetic parameters can depend on the environment. The MTRN model was, therefore, more coherent from a technical and biological point of view for the genetic evaluation of the present Nelore population. The response to selection should vary according to the environment considered, especially when selecting for PWG and PRODAM. For scrotal circumference (SC), the response to selection depends little on the production environment, since there is very little change in the genetic parameters across the environmental gradient. In this respect, genotype by environment interaction $(\mathrm{G} \times \mathrm{E})$ is more important for $\mathrm{PWG}$ and PRODAM than it is for SC and should be included in the genetic evaluation of these traits. The traits $\mathrm{PWG}$ and PRODAM can be considered plastic traits, whereas SC is poorly plastic. The genetic trends in individual animal slopes indicate that the population is moving towards greater plasticity. This could be a matter of concern for breeders, since greater plasticity seems to limit heritability and, consequently, the responses to selection. Further research in this field is warranted.

Acknowledgments We are grateful to CFM-Leachman Pecuária Ltda. for providing the data set. This work was funded by Fundação de Amparo à Pesquisa do Estado de Mato Grosso (FAPEMAT).

\section{References}

Cardoso FF, Tempelman RJ (2012) Linear reaction norm models for genetic merit prediction of Angus cattle under genotype by environment interaction. J Anim Sci 90:2130-2141 
Corrêa MBB, Dionello NJL, Cardoso FF (2009) Genotype by environment interaction characterization and model comparison for post weaning gain adjustment of Devon cattle via reaction norms. R Bras Zootec 38:1460-1467

de Jong G (1995) Phenotypic plasticity as a product of selection in a variable environment. Am Nat 145:493-512

Eler JP, Silva JAIIV, Evans JL, Ferraz JBS, Dias F, Golden BL (2004) Additive genetic relationships between heifer pregnancy and scrotal circumference in Nellore cattle. J Anim Sci 82:2519-2527

Eler JP, Ferraz JBS, Balieiro JCC, Mattos EC (2008) Genetic analysis of average annual productivity of Nellore breeding cows (COWPROD). Genet Mol Res 7:234-242

Falconer DS (1952) The problem of environment and selection. Am Nat $86: 293-298$

Kolmodin R, Bijma P (2004) Response to mass selection when the genotype by environment interaction is modelled as a linear reaction norm. Genet Sel Evol 36:435-454

Lopes JS, Rorato PRN, Weber T, Boligon AA, Comin JG, Dornelles MdA (2008) Genotype and environment interaction effect on weights at birth, 205 and 550 days of age of Nellore cattle in the South Region of Brazil. R Bras Zootec 37:54-60

Mattar M, Silva LOC, Alencar MM, Cardoso FF (2011) Genotype × environment interaction for long-yearling weight in Canchim cattle quantified by reaction norm analysis. J Anim Sci 89:2349-2355

Menéndez-Buxadera A, Mandonnet N (2006) The importance of the genotype $\times$ environment interaction for selection and breeding programmes in tropical conditions. CAB Rev: Perspect Agric Vet Sci Nutrition Natural Resources 1:026

Misztal I, Tsuruta S, Strabel T, Auvray B, Druet T, Lee DH (2002) BLUPF90 and related programs (BGF90). In: Proceedings of the 7th World Congress on Genetics Applied to Livestock Production, Montpellier, France, 19-23 August 2002
Morales R, Menéndez-Buxadera A, Avilés C, Molina A (2013) Direct and maternal genetic effects for preweaning growth in Retinta cattle estimated by a longitudinal approach throughout the calving trajectory of the cow. J Anim Breed Genet 130:425-434

Nephawe KA, Neser FWC, Roux CZ, Theron HE, van der Westhuizen J, Erasmus GJ (1999) Sire x ecological region interaction in Bonsmara cattle. S Afr J Anim Sci 29:189-201

Pégolo NT, Oliveira HN, Albuquerque LG, Bezerra LAF, Lôbo RB (2009) Genotype by environment interaction for 450-day weight of Nelore cattle analyzed by reaction norm models. Genet Mol Biol $32: 281-287$

Pigliucci M (2005) Evolution of phenotypic plasticity: where are we going now? Trends Ecol Evol 20:481-486

Santana ML Jr, Eler JP, Bignardi AB, Ferraz JBS (2013a) Genetic associations among average annual productivity, growth traits, and stayability: a parallel between Nelore and composite beef cattle. J Anim Sci 91:2566-2574

Santana ML Jr, Eler JP, Cardoso FF, Albuquerque LG, Ferraz JBS (2013b) Phenotypic plasticity of composite beef cattle performance using reaction norms model with unknown covariate. Animal 7: 202-210

Tonsor SJ, Elnaccash TW, Scheiner SM (2013) Developmental instability is genetically correlated with phenotypic plasticity, constraining heritability, and fitness. Evolution 67:2923-2935

Windig JJ, Calus MPL, Beerda B, Veerkamp RF (2006) Genetic correlations between milk production and health and fertility depending on herd environment. J Dairy Sci 89:1765-1775

Windig JJ, Mulder HA, Bohthe-Wilhelmus DI, Veerkamp RF (2011) Simultaneous estimation of genotype by environment interaction accounting for discrete and continuous environmental descriptors in Irish dairy cattle. J Dairy Sci 94:31373147 